\title{
A Comparison between Time-Frequency and Cepstral Feature Representations for the Classification of Seismic-Volcanic Signals
}

\author{
Paola Alexandra Castro-Cabrera ${ }^{1}$, Mauricio Orozco-Alzate ${ }^{1}$, Andrea Adami ${ }^{2}$, \\ Manuele Bicego ${ }^{2}$, John Makario Londoño-Bonilla ${ }^{3}$, \\ and Germán Castellanos-Domínguez ${ }^{1}$ \\ 1 Universidad Nacional de Colombia - Sede Manizales - Signal Processing and \\ Recognition Group - km 7 vía al Magdalena, Manizales, 170003 - Colombia \\ \{pacastroc, morozcoa, cgcastellanosd\}@unal.edu.co \\ 2 Università degli Studi di Verona - Dipartimento di Informatica - Ca' Vignal 2, \\ Strada le Grazie 15, Verona, 37134 - Italia \\ andrea.adami@studenti.univr.it, manuele.bicego@univr.it \\ 3 Servicio Geológico Colombiano - Observatorio Vulcanológico y Sismológico de \\ Manizales - Avenida 12 de Octubre No. 15 - 47, Manizales, 170001 - Colombia \\ jmakario@sgc.gov.co
}

\begin{abstract}
The analysis and classification of seismic patterns, which are typically registered as digital signals, can be used to monitor and understand the underlying geophysical phenomena beneath the volcanoes. In recent years, there has been an increasing interest in the development of automated systems for labeling those signals according to a number of pre-defined volcanic, tectonic and environmental classes. The first and crucial stage in the design of such systems is the definition or adoption of an appropriate representation of the raw seismic signals, in such a way that the subsequent stage — classification - is made easier or more accurate. This paper describes and discusses the most common representations that have been applied in the literature on classification of seismic-volcanic signals; namely, time-frequency features and cepstral coefficients. A comparative study of them is performed in terms of two criteria: (i) the leave-one-out nearest neighbor error, which provides a parameterless measure of the discriminative representational power and (ii) a visual examination of the representational quality via a scatter plot of the best three selected features.
\end{abstract}

Keywords: Feature-based representations, seismic-volcanic signals, pattern classification.

\section{Introduction}

The design of automated classification systems is of great interest in volcano monitoring in order to lighten the very time consuming task of distinguishing among several classes of seismic-volcanic signals. This possibility has recently

E. Bayro-Corrochano and E. Hancock (Eds.): CIARP 2014, LNCS 8827, pp. 440447 2014.

(C) Springer International Publishing Switzerland 2014 
attracted the attention of researchers and practitioners from both pattern recognition and volcano seismology fields. Several alternatives have been proposed and tried for each of the stages in a conventional pattern classification system (see Fig. 1): representation, adaptation and classification [1. In spite of that and particularly for representation, a comparative study of the different alternatives has not been yet undertaken.

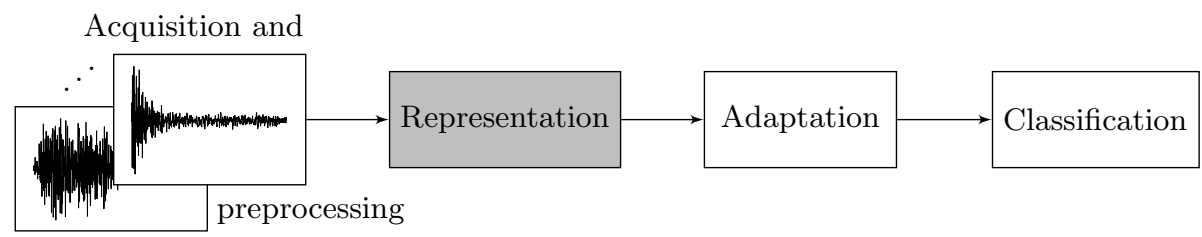

Fig. 1. Block diagram of a system for classifying seismic-volcanic signals

The importance of the representation stage has been clearly explained by Duin \& Pękalska in [2]: "The issue of representation is an essential aspect of pattern recognition [...]. It largely influences the success of the stages to come". The goodness of a representation can be judged by applying both quantitative and qualitative criteria. The first ones include - among others - interclass and intraclass distances, probabilistic distance measures and performance classification measures such as accuracies, confusion matrices and F-scores which are often preferred to be estimated for the one nearest neighbor $(1-N N)$ rule. The second ones encompass data visualizations such as scatter plots corresponding to data projections in $2 \mathrm{D}$ or $3 \mathrm{D}$ by applying, for instance, feature selection techniques.

This paper, therefore, is aimed to briefly describe the different representations for seismic-volcanic signals that are spread in the literature as well as to evaluate them under the same conditions and in terms of some of the above-mentioned criteria. A data set of labeled seismic signals from Nevado del Ruiz volcano (NRV) - Colombia, containing hundreds of examples per five seismic-volcanic classes, is used in the experiments.

\section{Methods}

\subsection{Generation of Feature Representations}

We compare four different representations in order to identify the one with the highest discriminant power over seismic-volcanic signals.

The $1^{\text {st }}$ set of features is the one presented by Curilem et al. in [3, where the following eight features were proposed. Four features are computed from the absolute value of the signal: 1) standard deviation, 2) mean, 3) median, and 4) maximum value. Two features, 5) kurtosis and 6) skewness, describe the sharpness and shape of the recording; they are obtained from the histogram with 200 bins. The two remaining features are based on the spectral content. The 
seventh feature gives a representative value for the frequency content of the signal and corresponds to 7) the mean frequency of the five highest peaks in its Fourier transform. The last feature is 8) the energy in a frequency sub-band. This energy is calculated from the coefficients obtained by a discrete wavelet decomposition in 5 frequency levels, whose frequency bands are below $1.5625 \mathrm{~Hz}$. The authors proposed this frequency because, according to their experience, it allows a better differentiation between long period seismic events and tremors (coming from seismic-volcanic events). The chosen mother wavelet was the daubechies 7 due to its similarity with the seismic-volcanic signal waveform. Finally, the feature vector obtained is normalized to the interval $\left[\begin{array}{ll}-1 & 1\end{array}\right]$.

The $2^{\text {nd }}$ set of features was originally proposed by Ibs-von Seht in 4 ; they describe waveform and spectrogram properties of seismic events by reading magnitudes on the time domain and characterizing the signal in the frequency domain by means of visual-defined patterns from its corresponding spectrogram. The features in this set are: 1) The event duration after raw signal segmentation. 2) The signal impulsiveness, which is a measure of how rapidly the signal amplitude has risen at the beginning of the event, it is computed as follows: the cumulative sum of signal absolute value $\sigma(i)$ is calculated with $i=1, \ldots, n-1$, where $n$ is the sample index of the signal. Then, it is determined the index $i_{c}$ where $\sigma(i)$ begins to be greater than $\sigma(i) * 2$; thereby, impulsiveness is defined as $i_{c} / n$. 3) Dominant frequency of the seismic event, defined as the number of zero crossings. The following features are calculated from the spectrogram: 4) The difference between the greatest and lowest frequencies of the spectral contour. 5) The frequency corresponding to the spectral contour centroid. 6) The frequency of the energy maximum in the spectrogram. Feature vectors are standardized (z-score) in order to guarantee a uniform influence of them during classification.

A $3^{\text {rd }}$ representation for seismic recordings is based on the approach proposed by Álvarez et al. in [5], in which they use speech recognition principles such as the short-time spectral envelop representation of the signal by means of MelFrequency Cepstral Coefficients (MFCC). That work contemplates the contributions made by the authors in previous publications, where different experimental configurations of MFCC were tested. The procedure applied to calculate such coefficients is not described here but can be found in [5] and references therein. In summary, the $3^{r d}$ feature set consists of a base vector of 13 coefficients (12 cepstral coefficients and the frame log-energy) plus their first order time derivatives, calculated to take into account the frame information.

The $4^{\text {th }}$ feature set has been recently applied in the seismic signal representation (e.g. in 6 6 7]). It consists in computing the spectrogram for each signal by means of the FFT and windowing with a defined overlap; then, an average of the spectrogram is calculated. For our experiments, the set of parameters were: a 128-point FFT, a 64-point Hamming window and an overlap of $50 \%$. Thereby, the feature vector generated by this representation corresponds to the mean value of each frequency band of the spectrogram across the time. 


\subsection{Discriminant Feature Selection}

Once the Representation stage is completed, a feature vector is obtained for each example, which, depending on the employed representation type, could have a large dimension; in consequence, many of its elements may be redundant or even irrelevant for the classification process. For such cases, adapting (see Fig. 1 again) the representation by applying, for example, dimensionality reduction techniques is suggested in order to find the most discriminant features as well as to avoid the "curse of dimensionality". Hence, feature selection algorithms can be applied over the whole feature space to determine the best feature subset for some criterion, by varying the number of selected features from 1 to $n$, where $n$ is the feature vector dimension. In this study, the well-known forward search algorithm was employed, using leave-one-out cross-validation error as evaluation criterion.

\subsection{Classification and Evaluation}

Most classifiers must be trained (parameter tunning) in order to maximize its performance; however, since our aim is testing the goodness of different representations, we restrict ourselves to a parameterless option: the 1- $N N$ rule.

Considering that our study is about a multiclass problem, evaluation of classification performance was carried out with measures which allow evaluate the performance per class, namely: confusion matrix , precision, recall and F1 scores; furthermore, accuracy and its standard error is reported as a global measure. Leave-one-out cross-validation was considered in order to make reproducible experiments with consistent results.

\section{Data Set Description: Seismic-Volcanic Classes, Signal Acquisition and Preprocessing}

The data set employed in this work consists of the four types of volcanic earthquakes typically defined by the volcanic seismology: volcano-tectonic (VT) events, long period (LP) events, tremors (TR), and hybrid (HB) events, all of them coming from Nevado del Ruiz volcano (NRV). Descriptions of their geophysical nature can be easily found in the volcano seismology literature. In addition to them, a fifth class - Tornillo (TO) events - is included in our study because they are commonly registered in NRV.

The data were acquired by the station named Bis, which is provided with a short period seismometer of three components that uses a 16-bit analog to digital converter with a sampling rate of $100 \mathrm{~Hz}$. In our study, only recordings of the vertical component were employed.

Recordings are manually segmented by experts from Volcanological and Seismological Observatory at Manizales (OVSM) who stamp the beginning of the event ( $\mathrm{P}$ wave) and duration (coda) through a primary processing software. Class labels are also assigned by hand to each recording, choosing only one from the above-mentioned seismic categories. 
For this study, examples of the most representative seismic signals that characterize the activity of NRV were used as event classes, namely: VT (153), LP (333), TR (242), TO (104), and HB (393) 1 Employed data set contains 1225 segmented recordings with durations between 5.5 and $2442 \mathrm{~s}$ that correspond to the volcanic activity from January 2010 to September 2013.

\section{Experimental Results and Discussion}

The four feature representations described in Sec. 2.1 were applied to the data set; additionally, a $5^{\text {th }}$ feature set generated by combination (concatenation) of them was analyzed. Their discrimination power was tested by using the $1-N N$ classifier.

Table 1 compiles the confusion matrices and other classification performance measures per class defined for the feature sets. An analysis per class of the achieved results indicates that for $\mathrm{HB}$ class, although $3^{\text {rd }}$ and $4^{\text {th }}$ representations show similar performances, the latter could be more appropriate because it obtained more true positives; however, that representation is more susceptible to confuse HB with LP events. Regarding LP class, it can also be well represented by $3^{\text {rd }}$ or $4^{\text {th }}$ feature set but, as in the previous case, more LP examples are confused as $\mathrm{HB}$ events with the $4^{\text {th }}$ representation than with the $3^{\text {rd }}$ one. This fact can be explained by the combined nature of hybrid events, which implies both rock fracture (typical VT mechanism) and fluid movements (LP mechanism) for their generation.

Regarding the TO class, a better classification performance for it was shown with the $3^{r d}$ representation, whereas TR class obtained its best classification performance with the $4^{\text {th }}$ representation. It is remarkable that VT is the class with less missclassifications, fact that is noticed with the overall highest precision, recall and F1 score figures achieved for this class when using the $4^{\text {th }}$ representation set, due to its well defined spectral components and characteristic waveform.

In general, according to the accuracy values and the standard errors obtained for the four representations (namely: 0,7061 $\pm 0,0130 ; 0,7143 \pm 0,0129 ; 0,7829 \pm$ 0,0118 and $0,8073 \pm 0,0113$, in their respective order), it is noticed that the averaged spectrogram-based representation leads to a better identification of the seismic-volcanic events with respect to the other three representations.

The feature set comprised by the four concatenated representations obtained a classification performance (see Table 2. part (a)) comparable with the one achieved by the $4^{\text {th }}$ representation, which was considered as the best in terms of accuracy and standard error $(0,8073 \pm 0,0113$ vs $0,8065 \pm 0,0113)$. The goodness of the $4^{\text {th }}$ representation was slightly affected by the redundant information contributed by the remaining features, leading to the requirement of a feature selection in order to identify the most discriminant ones.

After applying a standard selection algorithm (forward search) over the concatenated featured set, the dimensionality was reduced from 105 to 57, which represents a $46 \%$ less features to process in the subsequent stage; this is reflected

\footnotetext{
${ }^{1}$ The number of examples per class is given between parentheses.
} 
Table 1. Confusion matrices and performance measures for the four representations sets under comparison

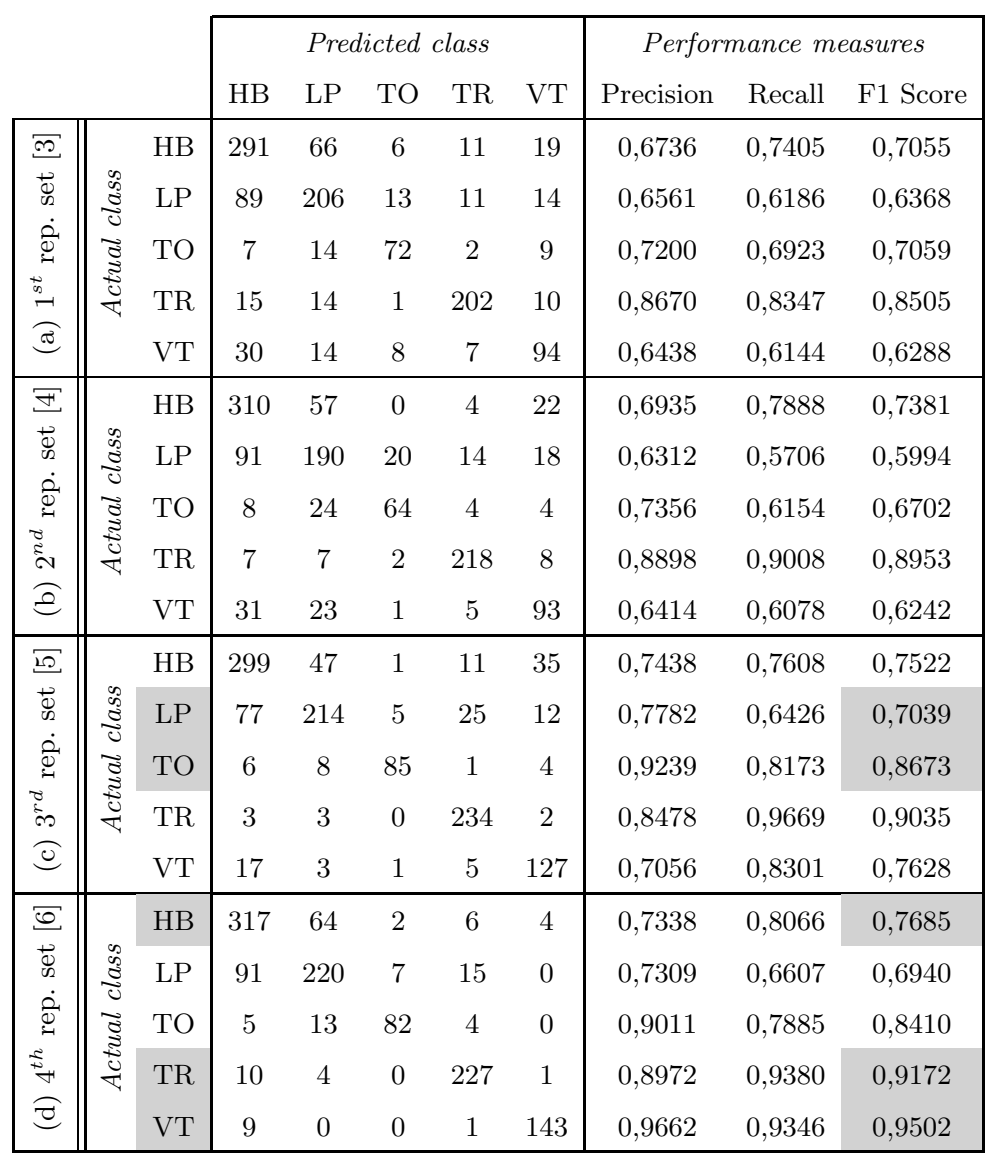

in a considerable computational cost reduction for classification. In addition, the accuracy $(0,8637 \pm 0.0098)$ was the highest one achieved among all representations; the same behavior is observed for the other performance measures for each class, as shown in part (b) of Table 2 .

In the resulting feature subset, $40 \%$ of the features belong to $4^{\text {th }}$ representation and $35 \%$ to the $3^{\text {rd }}$ one, which explains why those two feature sets give a better representation for all classes. Accordingly, the three most discriminant features identified by the selection algorithm are: the frequency corresponding to the spectral contour centroid (from $2^{\text {nd }}$ representation), the first order derivative of the first MFCC (from $3^{r d}$ representation) and the standard deviation of the seismic signal (from $1^{\text {st }}$ representation). These three features were used to generate a space in which the examples were placed in order to give a $3 \mathrm{D}$ visualization and examine the compactness or dispersion of the classes; notice 
Table 2. Confusion matrices and performance measures for (a) combination/concatenation of the four representation sets and (b) best feature subset selected by forward selection

\begin{tabular}{|c|c|c|c|c|c|c|c|c|c|c|}
\hline & & \multicolumn{5}{|c|}{ Predicted class } & \multicolumn{3}{|c|}{ Performance measures } \\
\hline & & & $\mathrm{HB}$ & LP & TO & TR & VT & Precision & Recall & F1 Score \\
\hline \multirow{5}{*}{ 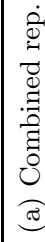 } & \multirow{5}{*}{ 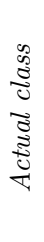 } & $\mathrm{HB}$ & 312 & 67 & 2 & 7 & 5 & 0,7446 & 0,7939 & 0,7685 \\
\hline & & LP & 86 & 220 & 8 & 18 & 1 & 0,7261 & 0,6607 & 0,6918 \\
\hline & & TO & 5 & 13 & 83 & 3 & 0 & 0,8830 & 0,7981 & 0,8384 \\
\hline & & TR & 8 & 3 & 0 & 230 & 1 & 0,8880 & 0,9504 & 0,9182 \\
\hline & & VT & 8 & 0 & 1 & 1 & 143 & 0,9533 & 0,9346 & 0,9439 \\
\hline \multirow{5}{*}{ 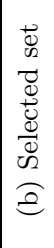 } & \multirow{5}{*}{ 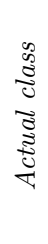 } & HB & 336 & 49 & 0 & 7 & 1 & 0,8175 & 0,8550 & 0,8358 \\
\hline & & LP & 58 & 259 & 3 & 11 & 2 & 0,8069 & 0,7778 & 0,7920 \\
\hline & & TO & 7 & 9 & 84 & 4 & 0 & 0,9655 & 0,8077 & 0,8796 \\
\hline & & TR & 6 & 4 & 0 & 231 & 1 & 0,9094 & 0,9545 & 0,9315 \\
\hline & & VT & 4 & 0 & 0 & 1 & 148 & 0,9737 & 0,9673 & 0,9705 \\
\hline
\end{tabular}

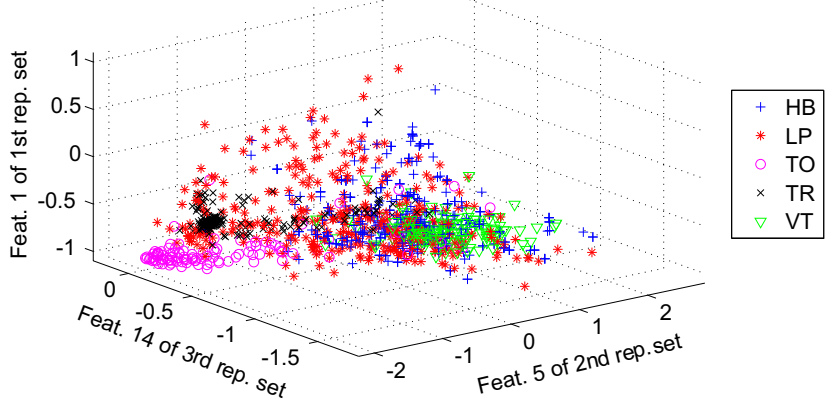

Fig. 2. Scatter plot of the data set. Axes correspond to the best three features selected from the concatenated feature sets.

in Fig. 2 that TO and TR classes are the more compact groups (although with outliers); consequently, they are the classes with less false negatives.

\section{Conclusion}

A proper pattern representation is crucial to achieve an optimal performance in an automatic recognition system. In the case of seismic-volcanic signals, a considerable number of representations have been proposed, however, a direct comparison of them had not been given. In this paper, a comparison of four representations mainly based on spectral and morphological features has been 
presented. Performance measures for the $1-N N$ rule showed that the best signal representation is the one based on averaged spectrograms ( $4^{\text {th }}$ representation), achieving an accuracy and standard error of $0,8073 \pm 0,0113$; furthermore, with this feature set, VT class obtained the highest accuracy rate with respect to the other classes. In addition, a selection of the most discriminant features indicated that 1 ) the frequency corresponding to the spectral contour centroid, 2) the first order derivative of the first MFCC and 3) the standard deviation of the seismic signal, provide an acceptable representation. As future work, the evaluation of features recently proposed in [8] is already under consideration.

Acknowledgments. The authors would like to thank Programa de Formación Doctoral en Colombia - Becas COLCIENCIAS for supporting this work, and also the staff members from the OVSM for providing the data set.

\section{References}

1. Orozco-Alzate, M., Acosta-Muñoz, C., Londoño-Bonilla, J.M.: The Automated Identification of Volcanic Earthquakes: Concepts, Applications and Challenges. In: D'Amico, S. (ed.) Earthquake Research and Analysis - Seismology. Seismotectonic and Earthquake Geology, pp. 345-370. InTech (2012)

2. Duin, R.P.W.: The science of pattern recognition. Achievements and perspectives. In: Duch, W., Mańdziuk, J. (eds.) Challenges for Computational Intelligence. SCI, vol. 63, pp. 221-259. Springer, Heidelberg (2007)

3. Curilem, G., Vergara, J., Fuentealba, G., Acuña, G., Chacón, M.: Classification of seismic signals at Villarrica volcano (Chile) using neural networks and genetic algorithms. J. of Volcanol. and Geotherm. Res. 180(1), 1-8 (2009)

4. Ibs-von Seht, M.: Detection and identification of seismic signals recorded at Krakatau volcano (Indonesia) using artificial neural networks. J. of Volcanol. and Geotherm. Res. 176(4), 448-456 (2008)

5. Álvarez, I., García, L., Cortés, G., Benítez, C., de la Torre, Á.: Discriminative Feature Selection for Automatic Classification of Volcano-Seismic Signals. IEEE Geosci. and Remote Sens. Lett. 9(2), 151-155 (2012)

6. Avesani, R., Azzoni, A., Bicego, M., Orozco-Alzate, M.: Automatic classification of volcanic earthquakes in HMM-induced vector spaces. In: Alvarez, L., Mejail, M., Gomez, L., Jacobo, J. (eds.) CIARP 2012. LNCS, vol. 7441, pp. 640-647. Springer, Heidelberg (2012)

7. Bicego, M., Acosta-Muñoz, C., Orozco-Alzate, M.: Classification of seismic volcanic signals using hidden-Markov-model-based generative embeddings. IEEE Trans. Geosci. Remote Sens. 51(6-1), 3400-3409 (2013)

8. Cortés, G., García, L., Álvarez, I., Benítez, C., De la Torre, Á., Ibañez, J.: Parallel system architecture (PSA): An efficient approach for automatic recognition of volcano-seismic events. J. of Volcanol. and Geotherm. Res. 271, 1-10 (2014) 